THE ASTROPHYSICAL JOURNAL, 565:125-130, 2002 January 20

(C) 2002. The American Astronomical Society. All rights reserved. Printed in U.S.A.

\title{
FILAMENTS AND IONIZED GAS IN THE VICINITY OF 3C $244.1^{1}$
}

\author{
CARlos FEINSTEIN \\ Observatorio Astronómico, Paseo del Bosque, 1900 La Plata, Argentina; and Space Telescope Science Institute, \\ 3700 San Martin Drive, Baltimore, MD 21218; cfeinstein@fcaglp.edu.ar \\ F. Duccio MACCheTto ${ }^{2}$ \\ Space Telescope Science Institute, 3700 San Martin Drive, Baltimore, MD 21218 \\ ANDRÉ R. MARTEL \\ Department of Physics and Astronomy, Johns Hopkins University, 3400 North Charles Street, Baltimore, MD 21218 \\ AND \\ William B. Sparks \\ Space Telescope Science Institute, 3700 San Martin Drive, Baltimore, MD 21218 \\ Received 2001 August 29 ; accepted 2001 September 26
}

\begin{abstract}
We present results of Hubble Space Telescope observations of the radio galaxy 3C 244.1. The broadband F702W $(R)$ and F555W $(V)$ images (WFPC2/PC) show an elliptical galaxy, and gaseous filaments and blobs surrounding it. In the narrowband ramp filter, dominated by $[\mathrm{O}$ III $] \lambda 5007$, these filaments are bright and have the same morphology as the broadband images. To the south, the filaments have a cone-shaped structure, and the radio jet is located at the center of this cone. To the north of the galaxy, the structure is found near the nucleus of the galaxy within its elliptical profile. From the photometry, the two brighter structures seem to be extended narrow-line emission regions. A comparison with diagnostic line ratios shows that the observed emission is consistent with interactions between the expanding radio jet and the local denser medium.
\end{abstract}

Subject headings: galaxies: active - galaxies: individual (3C 244.1) - galaxies: jets

\section{INTRODUCTION}

The Hubble Space Telescope (HST) has been used to undertake a systematic survey of extragalactic radio sources, the 3CR Snapshot Survey (de Koff et al. 1996; Martel et al. 1998, 1999 ; McCarthy et al. 1997; de Vries et al. 1997), selected from the Revised Third Cambridge Catalogue of Radio Sources (3CR; Bennett 1962a, 1962b; Spinrad et al. 1985). These data, taken with the Wide Field Planetary Camera (WFPC2) using the F702W, F555W, and ramp narrowband filters, allow us to investigate the relationships between the radio and optical morphologies in a large sample of powerful radio galaxies over redshifts of $0<z<1.5$.

Imaging with the HST of nearby Seyfert galaxies, such as Mrk 3, Mrk 6, Mrk 573, NGC 1068, NGC 2992 and NGC 4151 (Capetti et al. 1995a, 1995b, 1996; Capetti, Axon, \& Macchetto 1997; Winge et al. 1997; Allen 1998; Allen et al. 1999; Axon et al. 1998), has shown an intimate connection between radio structure and extended narrow-line regions (ENLRs). These studies show that the interaction of the radio jet with the interstellar medium (ISM) is the main source of UV photons that ionize the ENLRs in Seyfert galaxies. In previous work using HST data of 3C 299, Feinstein et al. (1999) found that the northeast radio lobe lies within a shell-like structure of the ENLR, suggesting a physical connection between the jet and this ENLR. Evidence of this interaction comes from the values of the line ratio diagnostics and the $[\mathrm{O} \mathrm{II}] \lambda 3727 /[\mathrm{O} \mathrm{III}] \lambda 5007$ ratio, an estimator of the change of the ionization parameter $U$ over the ENLR. We are extending this work by investigat-

\footnotetext{
${ }^{1}$ Based on observations made with the NASA/ESA Hubble Space Telescope, which is operated by the Association of Universities for Research in Astronomy, Inc., under NASA contract NAS 5-26555.

${ }^{2}$ On assignment from the Space Science Department of ESA.
}

ing whether this scenario is also applicable to other powerful radio sources. In this paper, we discuss the radio galaxy 3C 244.1

3C 244.1 is an FR II radio source with two lobes with an angular separation of 52" at a position angle (P.A.) of $168^{\circ}$. Both lobes have the same flux density [Fernini, Burns, \& Perley 1997; $\left.\log \left(L_{5 \mathrm{GHz}}\right) \approx 22 \mathrm{~W} \mathrm{~Hz}{ }^{-1}\right]$. Fernini et al. (1997) also detected the central component of the source that coincides with the optical identification of Spinrad et al. (1985), showing that the radio lobes are located asymmetrically with respect to this central compact source.

At optical wavelengths, the host galaxy of 3C 244.1 was shown to have strong emission lines and a redshift of $z=0.428$ (Spinrad et al. 1985). From CCD images, McCarthy, Spinrad, \& Van Breugel (1995) note that the galaxy lies in a cluster, and their [O III] image shows a high surface brightness fan of emission extending $\sim 7^{\prime \prime}$ to the northwest, with a P.A. similar to the radio structure.

In this paper we discuss the morphology of 3C 244.1 as shown by the high spatial resolution images of WFPC2, and we investigate the possible scenarios that give rise to the observed emission morphology.

\section{OBSERVATIONS AND DATA ANALYSIS}

The HST/WFPC2 observations of 3C 244.1 were taken as part of the 3CR imaging Snapshot Survey (PI W. B. Sparks), which was conducted in Cycles 4-8 in the F555W and $\mathrm{F} 702 \mathrm{~W}$ broad bands and narrow emission-line bands (de Koff et al. 1996; Martel et al. 1998, 1999; McCarthy et al. 1997; etc.). The 3C 244.1 images were obtained with the WFPC2/PC in 1994 April and 1995 May for the F702W filter and in 1996 July for the F555W filter. Narrowband images with the WFPC2/WF2 and the FR680N ramp filter were taken in 1995 November. Table 1 shows the observation $\log$. 
TABLE 1

LOG OF OBSERVATIONS

\begin{tabular}{|c|c|c|c|}
\hline Filter Name & Emission Line & $\begin{array}{l}\text { Exposure Time } \\
\text { (s) }\end{array}$ & Date \\
\hline F702W . & Broadband $^{\text {a }}$ & 300 & 1994 Apr 17 \\
\hline F702W. & Broadband $^{a}$ & 300 & 1995 May 28 \\
\hline FR680N...... & {$[\mathrm{O} \mathrm{III}] \lambda 5007$} & 300 & 1995 Nov 13 \\
\hline FR680N...... & {$[\mathrm{O} \mathrm{III}] \lambda 5007$} & 300 & 1995 Nov 13 \\
\hline F555W ....... & Broadband $^{\mathrm{a}}$ & 300 & 1996 Sep 7 \\
\hline F555W ....... & Broadband $^{\mathrm{a}}$ & 300 & $1996 \mathrm{Sep} 7$ \\
\hline
\end{tabular}

${ }^{a}$ Due to the filter width, the measured flux includes emission from several lines; see text.

At a redshift of $z=0.428$, the galaxy is at a distance of 1.5 Gpc, with a projected linear scale of $5.1 \mathrm{kpc} \operatorname{arcsec}^{-1}$ (assuming $H_{0}=65 \mathrm{~km} \mathrm{~s}^{-1} \mathrm{Mpc}^{-1}$ and $q_{0}=0.5$ ). The WFPC2/PC scale is $0{ }^{\prime \prime} 0455$ pixel $^{-1}$, and for the WFPC2/ WF2, the scale is $0^{\prime \prime} 0996$ pixel $^{-1}$, and therefore, the physical scales for the images are $232 \mathrm{pc} \mathrm{pixel}^{-1}$ for the PC mode and $492 \mathrm{pc} \mathrm{pixel}^{-1}$ for the WF2 mode.

The reduction procedure for the data of the F702W filter, which is close to a Cousins $R$ filter, is fully discussed in Martel et al. (1999). This reduction includes the standard WFPC2 pipeline processing followed by cosmic-ray removal. The data reduction was carried out using IRAF and the STSDAS package. At the redshift of 3C 244.1, the F702W filter includes the $\mathrm{H} \gamma$, [O III] $\lambda 4363$, He II $\lambda 4686, \mathrm{H} \beta$, [O III] $\lambda \lambda 4959,5007$, and [N I] $\lambda 5199$ emission lines. The data of the F555W filter, which is close to Johnson $V$, was reduced in the same manner and then registered and added to produce one final image with higher $\mathrm{S} / \mathrm{N}$. Because of the redshift, the F555W filter includes the flux from the emission lines of $[\mathrm{Ne} \mathrm{v}] \lambda 3426,[\mathrm{O} \mathrm{II}] \lambda 3727,[\mathrm{Ne}$ III $] \lambda 3869, \mathrm{H} \delta$, and $\mathrm{H} \gamma$ (the latter considerably attenuated by the filter response). In what follows, we define F702W as $R$ and $\mathrm{F} 555 \mathrm{~W}$ as $V$. The FR680N is a narrowband ramp filter that, given the redshift of the galaxy and its position on the $\mathrm{CCD}$, produces an [O III] $\lambda 5007$ image. These images were reduced as described above, and the final image was rescaled to the resolution of the WFPC2/PC.

The F702W and F555W filter data were flux-calibrated using values for the inverse sensitivities (PHOTFLAM) of $1.965 \times 10^{-18}$ and $3.49141 \times 10^{-18} \mathrm{ergs} \mathrm{cm}^{-2} \AA^{-1} \mathrm{DN}^{-1}$, where DN stands for data number, and zero points for the Vega system of $M_{\text {photzpt }}=-21.85$ and -21.07 , respectively. Nonrotated images were used for these flux calibrations to minimize interpolation errors. The FR680N filter was calibrated using the ramp filter calculator (Biretta et al. 1996). An approximate coordinate frame for the WFPC2 data is provided by the image-header information, based on the HST guide stars. The accuracy for the coordinates is approximately 1" (Biretta et al. 1996).

Images obtained with different filters were registered to a common reference frame using cross-correlation of the brighter elliptical structures. The sky background in each image was determined through the statistical analysis of a 3 $\sigma$ clipping of the average background value in several regions in each of the images.

\section{RESULTS}

The optical broadband images show an elliptical galaxy with blobs and filaments (Figs. 1 and 2). Both the $V$ and $R$ images show these structures, and some are also present in

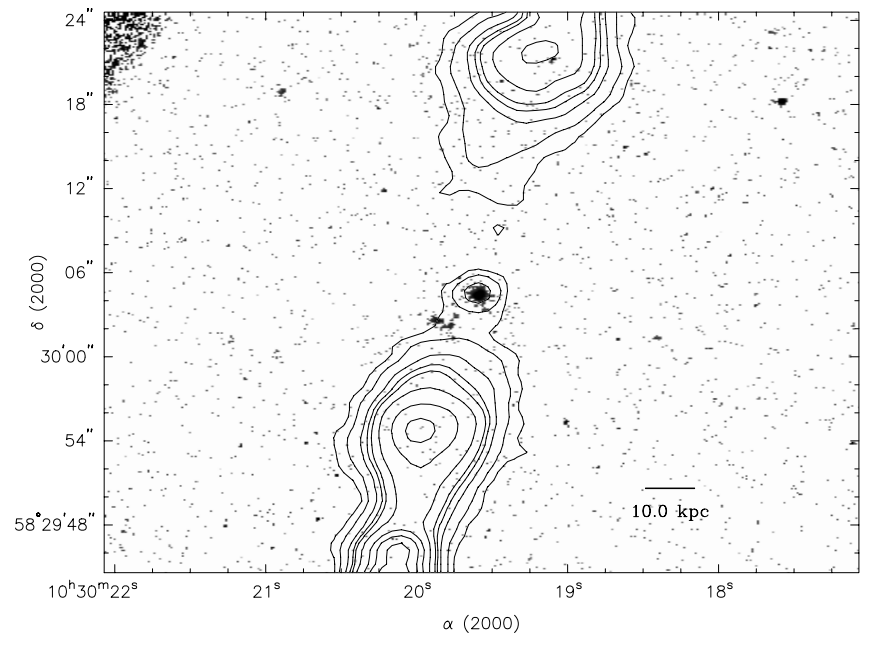

FIG. 1.-3C 244.1 $R$-band image taken with the WFPC2/F702W filter (gray scale). The contours are from the radio map (J. P. Leahy 1999, private communication).

the ramp image. We have named the most prominent of these structures A, B, C, D, and E (Fig. 3). To compare with radio data, we superimposed an $8.4 \mathrm{GHz}$ radio map from the observations of J. P. Leahy (1999, private communication) in Figure 1. The total extension of the radio structure is $51^{\prime \prime}$ (from lobe to lobe), larger than the size of these HST images.

The object named A is bright and extended and is located 2".9 from the nucleus (P.A. $125^{\circ}$ ), having the same shape in all filters. The flux in the narrow filter implies a large [O III] $\lambda 5007$ emission. The structure named B is located 2".6 away from the nucleus at P.A. $150^{\circ}$ and is similar in shape to structure A, albeit fainter. Both of these structures (A and B) seem to be connected to the galaxy by very faint filaments.

The object named $\mathrm{C}$ is located to the west, at a separation of 1 ."4 from the nucleus at P.A. $210^{\circ}$. A filament appears to connect it directly to the center of the galaxy. Superimposed over this line at $1^{\prime \prime}$ from the nucleus, there is another blob $\left(C^{\prime}\right)$. The filament just described and those associated with blobs A and B can be interpreted as the edges of a biconic structure with an opening angle of $85^{\circ}$. This kind of morphology has been found to be common in a variety of active galaxies ranging from nearby spirals (for example NGC 1068, Evans et al. 1991; NGC 5728, Wilson et al. 1993) to powerful 3CR objects (e.g., 3C 299 Feinstein et al. 1999). The southern radio jet is located in the middle of this biconical structure.

The structure D is located inside the elliptical profile of the galaxy, northeast of the nucleus. After the subtraction of an $r^{1 / 4}$ profile (using the IRAF/STSDAS task ELLIPSE) from the image, a filamentary link emerges connecting $D$ to the galaxy nucleus (see Fig. 1). This structure seems not to be as large as the others and is very difficult to measure in the broadband images because of the steep shape of the elliptical galaxy profile. This structure is also evident in the [O III] $\lambda 5007$ image, but as for the broadband data, it is not possible to measure its flux reliably.

The structure named E, at 3".3 from the nucleus at P.A. $110^{\circ}$, is not present in the narrowband filter. Its profile is compact and steep, showing all the typical characteristics of a field galaxy with a redshift different from that of 3C 244.1. 


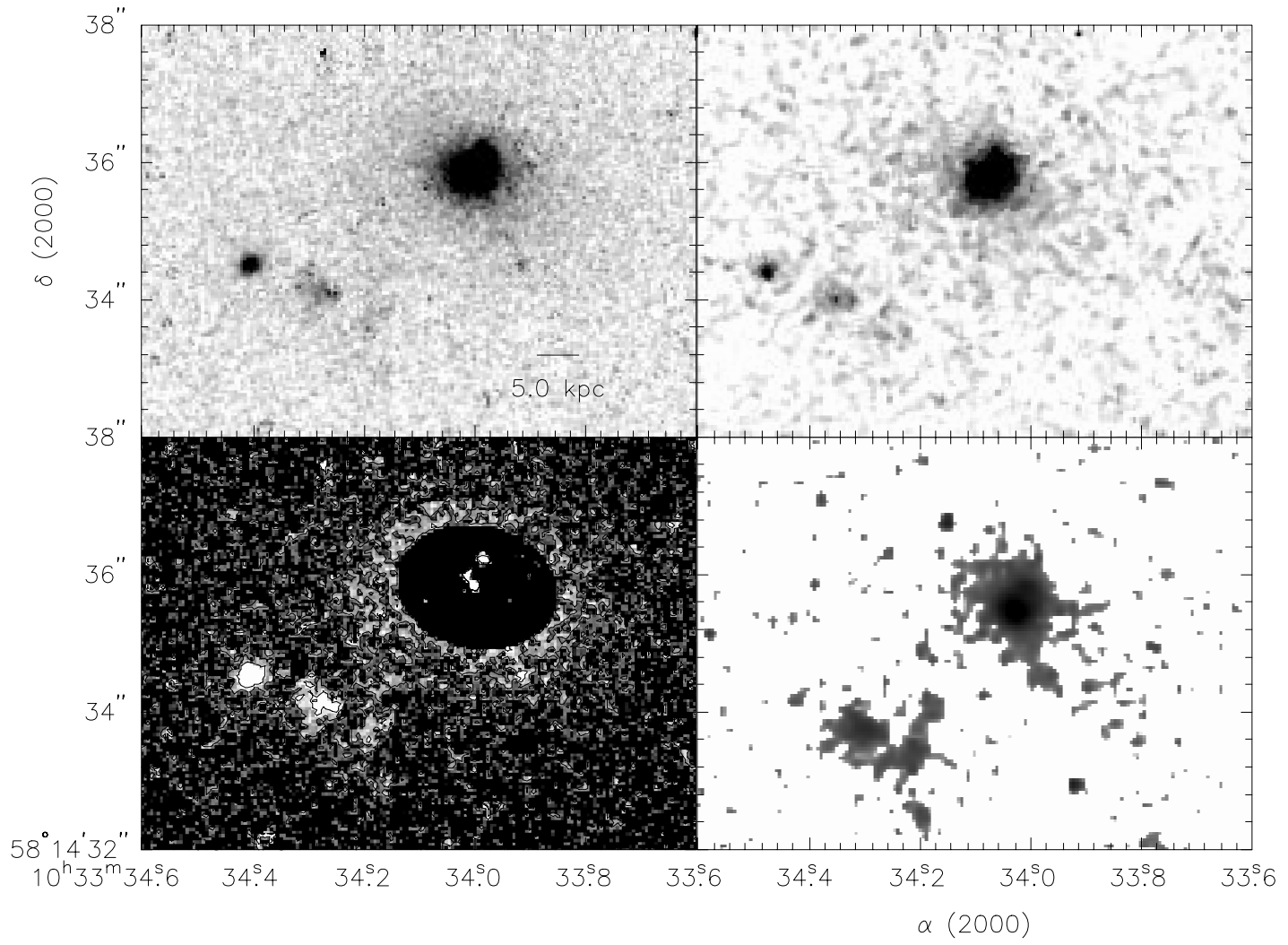

FIG. 2.-3C 244.1. Top left: $R$-band image (F702W). Top right: $V$-band image (F555W). Bottom left : $R$-band image with the elliptical profile subtracted (note the plume of emission to the northwest). Bottom right: $[\mathrm{O}$ III $] 25007$ (rescaled from the WFPC2/WF to the resolution of the WFPC2/PC).

We have carried out photometry of the structures surrounding $3 \mathrm{C} 244.1$ using the IRAF task POLYPHOT (from the NOAO APPHOT package). This IRAF task computes the magnitude of an object in an image inside a polygonal aperture. The sky background is also computed and subtracted, and the errors are calculated. To measure the bulk of the emission for each structure, a polygon that closely matched the shape of the region was carefully chosen. The same polygons were used on all images from the different filters to make the data comparable between these filters.

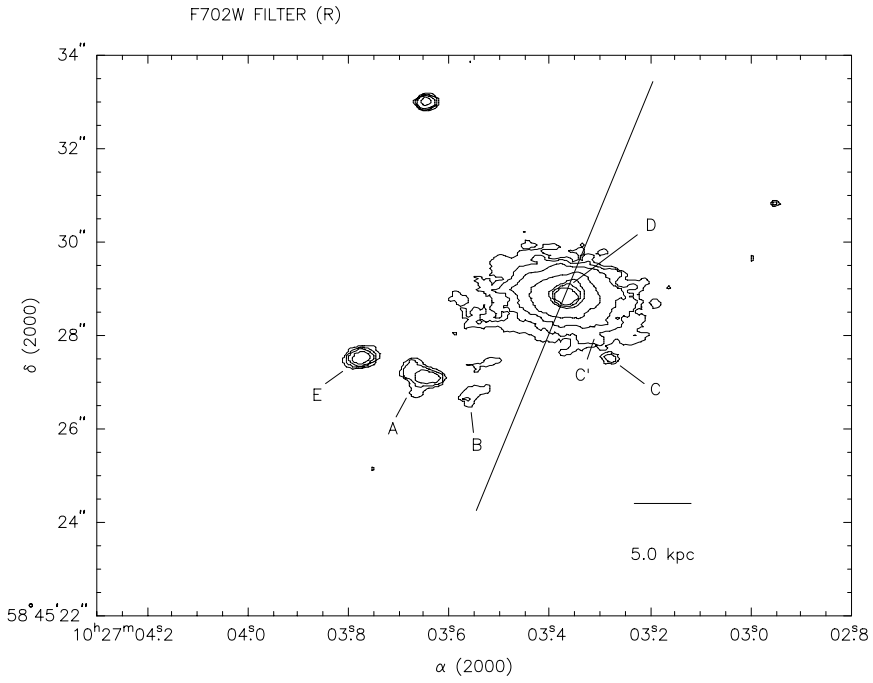

FIG. 3.- 3C $244.1 R$-band image with the identifications of the structures. The straight line indicates the direction of the radio jet.
However, for the $V$ filter, in which the signal was lower, some of these structures were difficult to measure above the background noise.

Table 2 shows the result of the photometry using the polygonal measurement technique and the subsequent calibration. For the broadband filters, the data were calibrated using the inverse sensitivity (PHOTFLAM) and the filter width (see the Wide Field and Planetary Camera Instrument Handbook; Biretta et al. 1996). For the FR680N filter, the calibration was done using the IRAF/STSDAS SYNPHOT, and checked with the WFPC2 Exposure Time Calculator (ETC). The flux observed at this filter, basically the flux of the [O III] $\lambda 5007$ line, was corrected by a small contamination $(\sim 12 \%)$ of the [O III] $\lambda 4959$ line (assuming that the flux of the [O III] $\lambda 5007$ line is 2.88 times the [O III] $\lambda 4959$ flux). This correction was calculated by computing the central wavelength at the position on the CCD, and assuming that the filter is Gaussian, centered at that wave-

TABLE 2

TOtAl FluX ${ }^{a}$ From the Structures

\begin{tabular}{ccccc}
\hline \hline $\begin{array}{c}\text { Region } \\
(1)\end{array}$ & $\begin{array}{c}\text { F702W } \\
(2)\end{array}$ & $\begin{array}{c}\text { F555W } \\
(3)\end{array}$ & $\begin{array}{c}\text { [O III] } \lambda 5007 \\
(4)\end{array}$ & $\begin{array}{c}\text { F702W }^{\mathrm{b}} \\
(5)\end{array}$ \\
\hline $\mathrm{A} \ldots \ldots$ & $17.26 \pm 1.37$ & $20.57 \pm 2.69$ & $9.36 \pm 1.24$ & $24.12 \pm 1.91$ \\
$\mathrm{~B} \ldots \ldots$. & $5.25 \pm 0.82$ & $5.04 \pm 1.62$ & $3.58 \pm 0.77$ & $7.34 \pm 1.14$ \\
$\mathrm{C} \ldots \ldots$ & $2.6 \pm 0.59$ & $\ldots$ & $\ldots$ & $3.62 \pm 0.83$ \\
$\mathrm{C}^{\prime} \ldots \ldots$ & $2.3 \pm 0.52$ & $\ldots$ & $\ldots$ & $3.24 \pm 0.74$ \\
\hline
\end{tabular}

Note-Measurements with values less than 3 times the error are not reported.

${ }^{a}$ Flux units are $10^{-16} \mathrm{ergs} \mathrm{cm}^{-2} \mathrm{~s}^{-1}$.

b The F702W filter flux is calibrated assuming that the flux distribution is completely dominated by emission lines. See text. 
TABLE 3

\begin{tabular}{|c|c|}
\hline Line & Quantum Efficiency \\
\hline $\mathrm{H} \gamma, \ldots \ldots \ldots \ldots \ldots$ & 0.133 \\
\hline$[\mathrm{O}$ III $] \lambda 4363 \ldots \ldots \ldots$ & 0.139 \\
\hline$[\mathrm{He}$ II $] \lambda 4686 \ldots \ldots \ldots$ & 0.132 \\
\hline $\mathrm{H} \beta \ldots \ldots \ldots \ldots \ldots$ & 0.124 \\
\hline$[\mathrm{O}$ III $] \lambda 4959 \ldots \ldots \ldots$ & 0.113 \\
\hline$[\mathrm{O}$ III $] \lambda 5007 \ldots \ldots \ldots$ & 0.112 \\
\hline$[\mathrm{N} \mathrm{I}] \lambda 5199 \quad \ldots \ldots \ldots .$. & 0.092 \\
\hline
\end{tabular}

length with a FWHM of $1.3 \%$ (value obtained from the ETC) of this central wavelength. Columns (2), (3), and (4) of Table 2 are the results of these calibrations.

From Figure 1, structures A and B are very close to the jet flow, so the first task was to test whether these objects were optical synchrotron knots on the radio jet. Therefore, we fitted a power law to the flux from the broadband filters F555W and F702W. If the main source of flux is synchrotron radiation, the flux must follow a power law with an exponent in the range -0.5 to -1.5 . To make the test as accurate as we could, we used the task CALCPHOTSYNPHOT (from the SYNPHOT/STSDAS IRAF package) to integrate the power law under a better simulation of each filter. We found that the observed flux is not a power law, and the best fit is a flat distribution with a null slope. Therefore, we conclude that the observed flux is inconsistent with power-law distributions of optical synchrotron radiation.

From the broadband filters, we can estimate the $(V-I)$ colors of the elliptical host galaxy A and B regions. Colors of the other structures cannot be measured unless we have a better model of the underlying galaxy flux, for which we need deeper observations and better spatial resolution. Objects A and B are far away from the elliptical profile and are clearly not contaminated. For the elliptical galaxy, we obtain $(V-I)=1.2 \pm 0.3$, which is close to the value predicted by Rönnback et al. (1996) for an elliptical galaxy at the redshift of 3C 244.1 $(z=0.428)$, while the objects A and $B$ seem to be bluer, with values of $-0.2 \pm 0.2$ and $-0.6 \pm 0.5$, respectively.

Both structures A and B, given the large flux in the narrow ramp filter, are obviously at the 3C 244.1 redshift. Also, both show the same morphology shape as the broadband images. Since the $R$-filter data also include the flux of $\mathrm{H} \gamma$, [O III $] \lambda 4363$, He II $\lambda 4686, \mathrm{H} \beta$, [O III] $\lambda \lambda 4959,5007$, and [N I] $\lambda 5199$ emission lines, the total flux measured in this broad band must be larger than the ramp filter, which only includes $[\mathrm{O}$ III $] \lambda 5007$. The fact that they are so similar can be explained if we assume that the flux originates in a pure emission-line region, without any significant continuum contribution.

As the narrowband filter data shows, there is a large amount of flux in the emission line [O III] 25007 . Thus, it is very reasonable to suggest that the structures $\mathrm{A}$ and $\mathrm{B}$ are ENLRs. The ENLRs have been found to be associated with a wide variety of active galactic nuclei (AGNs), from Seyfert 2 to radio galaxies.

Column (5) of Table 2 shows the flux calibration of the broadband $R$ filter, assuming that all the flux is from a source that has a spectrum dominated by bright emission lines. The shape of the F702W filter is practically flat (see Biretta et al. 1996), and so it is easy to compute the calibration of the flux of all the lines by calculating the total flux for only one emission line (that accounts for all the line emission flux). To make this calculation, the ETC was used, and the quantum efficiencies (system + filter + CCD, hereafter QT) for each wavelength of the conspicuous emission lines included in the $R$-filter band were computed. Table 3 shows the results of this calculation and confirms that all these emission lines are practically attenuated by similar amounts. Therefore, we used $\mathrm{H} \beta$ as an equivalent line (i.e., a line that has all the flux of all the lines included in the filter) for the calibration. The error of this procedure is extremely small.

\section{DISCUSSION}

A key question is to identify the mechanism responsible for the ionization of these structures and filaments. The most likely mechanisms are photoionization by the AGN, photoionization by the AGN with matter-bounded clouds, and shock ionization by the radio jet. We discuss each of these mechanisms in detail.

\subsection{Photoionization by the AGN}

This mechanism assumes that the UV flux produced by the AGN's nuclear source photoionizes the line-emission region. The physical status of the gas at any place can be described by the ionizing parameter $U$, which accounts for the dilution of the UV photons as the distance from the nucleus increases. For regions A and B, it is very unlikely that the AGN is the only source of photons, because of the large distances. To account for this scenario, some reference numbers of the total photon budget can be calculated.

Rawlings et al. (1989) performed spectroscopy of the nuclear zone of $3 \mathrm{C} 244.1$ and show a relation of [O III] $\lambda 5007 / \mathrm{H} \beta=4.5$. Using the results of Ferland \& Netzer (1983), which assume that the main source of photoionization continuum is a power law $\left(f_{v} \propto v^{-1.5}\right)$ and that the gas has a density of $N_{\mathrm{H}}=10^{3}$, they calculate several line emission ratios. Using these results, it is straightforward to compute the ionization parameter $U$. This leads to $U=3.2 \times 10^{-4}$ for solar abundances and $6.3 \times 10^{-4}$ for subsolar abundances. This spectrum covers only light coming from the nuclear zone that is observed in the narrowband filter (regions A and B are not included in this spectrum), so it is possible to calculate the total UV photon emission of the nucleus. Because $Q=U 4 \pi r^{2} N_{e} c$ and $r$ can be estimated from the WFPC2 emission-line image, this gives $Q=4.5 \times 10^{54}$ photons s $^{-1}$ for solar abundances and $9 \times 10^{54}$ photons s ${ }^{-1}$ for subsolar abundances. Due to the large distances, these two values (for each of these different abundances) are not enough to ionize the blobs A and B. Therefore, we conclude that direct photoionization by the nucleus is unlikely to be the dominant mechanism responsible for the observed ENLR emission.

\subsection{Photoionization by the AGN with Matter-bounded Clouds}

This idea was developed by Binette, Wilson, \& StorchiBergmann (1996) in order to explain the discrepant high ionization line ratios and high electron temperatures observed in many active galaxies. These models are based on a parameter $A_{M / I}$, which is the solid angle covered by the 
TABLE 4

Photolonization by an AGN with Matter-Bounded Clouds

\begin{tabular}{ccccc}
\hline \hline$A_{M / I}$ & $\begin{array}{c}\text { Model A } \\
U=0.04\end{array}$ & $\begin{array}{c}\text { Model H } \\
U=0.5\end{array}$ & $\begin{array}{c}\text { Model M } \\
U=0.05\end{array}$ & $\begin{array}{c}\text { Model L } \\
U=0.02\end{array}$ \\
\hline $0.04 \ldots \ldots$ & 1.79 & 1.83 & 4.95 & 6.77 \\
$0.14 \ldots \ldots$ & 1.74 & 1.76 & 2.99 & 3.19 \\
$0.24 \ldots \ldots$ & 1.71 & 1.71 & 2.46 & 2.50 \\
$0.34 \ldots \ldots$ & 1.68 & 1.67 & 2.21 & 2.21 \\
$0.44 \ldots \ldots$ & 1.66 & 1.65 & 2.06 & 2.05 \\
$0.54 \ldots \ldots$ & 1.65 & 1.63 & 1.97 & 1.95 \\
$0.64 \ldots \ldots$ & 1.64 & 1.61 & 1.90 & 1.88 \\
$0.74 \ldots \ldots$ & 1.63 & 1.60 & 1.85 & 1.83 \\
$0.84 \ldots \ldots$ & 1.62 & 1.59 & 1.81 & 1.79 \\
$0.94 \ldots \ldots$ & 1.61 & 1.58 & 1.78 & 1.76 \\
$1.04 \ldots \ldots$ & 1.60 & 1.57 & 1.76 & 1.73 \\
\hline
\end{tabular}

${ }^{a}$ Model from Binette et al. 1996, no special name assigned in the original paper. For other models of Binette et al. 1997, names are as in the original paper.

"matter-bounded" component relative to that covered by the "ionization-bounded" component. In another paper, Binette et al. (1997) refined their models. Taking into account the possibility of having a larger $U$ parameter, they derived three models with values of $U=0.5,0.05$, and 0.02 and a density $n_{e}=1000 \mathrm{~cm}^{-3}$ for the "matter bounded" clouds, which are exposed to ionization radiation from the AGN.

As we had only one line and the integrated total flux of several lines from the $R$ filter, we computed the flux ratio $C_{D}=R /[\mathrm{O} \mathrm{III}] \lambda 5007$, noting that the bulk of the $R$-filter flux is the sum of $\mathrm{H} \gamma$, [O III] $\lambda 4363, \mathrm{He}$ II $\lambda 4686, \mathrm{H} \beta$, [O III] $\lambda \lambda 4959,5007$, and $[\mathrm{N}$ I $] \lambda 5199$ fluxes. Note that the contribution from the stellar host galaxy is not significant at the location of the blobs. The line ratio can be derived from Table 2 and is $C_{D}=2.6$ for structure A and 2.0 for structure B.

We computed $C_{D}$ (the ratio of lines included in the $R$ filter to [O III] 25007) for the results of mixed models of the papers mentioned above. Table 4 shows these results of $C_{D}$ as a function of the $A_{M / I}$ parameter for the model of Binette et al. (1996) and models H, M, and L of Binette et al. (1997). Models $\mathrm{M}$ and $\mathrm{L}$ seem to fit the observed $C_{D}$ for structures $\mathrm{A}$ and $\mathrm{B}$, for $A_{M / I} \sim 0.2$ and 0.34 for each object, respectively. However, both of them have the same problem that we have discussed for the first case of photoionization by the AGN: $U=0.05$ for Model $\mathrm{M}$ and 0.02 for model $\mathrm{L}$, values that imply a very high $Q\left(Q=c n_{e} U d^{2}\right)$ due to the distances to the galaxy nucleus. This calculation makes the mixed models very unlikely.

TABLE 5

SHOCK MODELS

\begin{tabular}{cc}
\hline \hline $\begin{array}{c}\text { Shock Velocity } \\
\left(\mathrm{km} \mathrm{s}^{-1}\right)\end{array}$ & $C_{D}$ \\
\hline $150 \ldots \ldots \ldots \ldots \ldots \ldots \ldots$ & 2.29 \\
$200 \ldots \ldots \ldots \ldots \ldots \ldots$. & 2.67 \\
$200+$ Precursor $\ldots \ldots$. & 2.34 \\
$300 \ldots \ldots \ldots \ldots \ldots \ldots$. & 3.34 \\
$300+$ Precursor $\ldots \ldots$. & 1.67 \\
$500 \ldots \ldots \ldots \ldots \ldots \ldots$. & 2.71 \\
$500+$ Precursor $\ldots \ldots$. & 1.47 \\
\hline
\end{tabular}

\subsection{Shocks}

Figure 1 shows that the ENLR structures A and B are located close to the edges of the radio jet, and it is possible that there is an interaction between the jet and these regions. It is important to study whether there is a physical relationship between the radio jet and the optical line emission. Taylor, Dyson, \& Axon (1992) proposed that fast bow shocks resulting from the interaction of the radio jet and the ISM were the source of ionizing photons of the emissionline gas in a number of sources. Capetti et al. (1995a, 1995b, 1996, 1997) and Winge et al. (1997) were the first to show that this mechanism best explains the optical emission in the NLR in nearby Seyfert galaxies (Mrk 3, Mrk 6, Mrk 573, NGC 1068, NGC 4151, and NGC 7319). Recently, this work has been confirmed by Aoki et al. (1999) and Kukula et al. (1999). In the case of the powerful radio galaxies of the 3CR catalog, Feinstein et al. (1999) showed that this interaction also occurs in 3C 299, where clearly the northeast radio jet and the ENLR have similar morphologies, and where there is further evidence of this interaction from the values of the different emission-line ratios and the evolution of the line ratio $[\mathrm{O} \mathrm{II}] \lambda 3727 /[\mathrm{O} \mathrm{III}] \lambda 5007$, as an estimator of the changes of $U$, over the region.

Dopita \& Sutherland $(1995,1996)$ have modeled in detail the ionization of the ENLR due to shocks. In one scenario, which has been shown to work for Seyfert galaxies, the radio jet interacts with the local ISM and shocks the gas. In this scenario, the hot postshock plasma gas produces photons that can diffuse upstream and downstream of the jet. Photons diffusing upstream can encounter the preshocked gas and produce an extensive precursor $\mathrm{H}$ II region, while those traveling downstream will influence the ionization and temperature structure of the recombination of the shock.

To check the validity of this interpretation, we use the line diagnostic test of Dopita \& Sutherland (1996). We apply the same procedure as the case above and use the flux ratio $C_{D}=R /[\mathrm{O} \mathrm{III}] \lambda 5007$. On the other hand, we can compute the value of this ratio for emission lines arising in a shocked ENLR. Using Table 1 of Dopita \& Sutherland (1996), we calculate the total integrated flux for the lines involved and the ratio to [O III] $\lambda 5007$ flux as $C_{D}=$ $\sum_{i} F_{i} Q T_{i}\left[([\mathrm{O} \mathrm{III}] \lambda 5007) Q T_{[\mathrm{O} \mathrm{ml}] 25007}\right]^{-1}$, where $i$ represents each of the lines mentioned above and $F_{i}$ their respective flux.

Table 5 shows the results of this computation for shock speeds of $100,150,200$, and $500 \mathrm{~km} \mathrm{~s}^{-1}$, with and without a precursor $\mathrm{H}$ iI region. From this table, the values observed for structures $\mathrm{A}\left(C_{D}=2.6\right)$ and $\mathrm{B}\left(C_{D}=2.0\right)$ are easily explained with shock models of $\sim 200 \mathrm{~km} \mathrm{~s}^{-1}$. We conclude that shock interactions between the expanding jet and the local denser medium are responsible for the observed ENLR emission in 3C 244.1

\section{CONCLUSIONS}

We have shown that radio galaxy 3C 244.1 has a filamentary structure and some blobs bright in emission line ([O III $]$ 25007). To the south, this structure seems to be an emission-line cone with an opening angle of $85^{\circ}$ (from P.A. $125^{\circ}$ to $210^{\circ}$ ). The radio jet is located at the center of this cone (P.A. $168^{\circ}$ ). The two brighter blobs (named A and B) are likely part of an ENLR (by comparing the flux between broad band and narrow band), similar to those associated 
with AGNs. These structures are larger and located far away from the nucleus (2".9). To the north, more of these structures were found, but close to and associated with the nucleus.

We tested several scenarios to explain the source of energy for the emission lines observed, and we found that direct photoionization by the AGN can only be possible if a large and unlikely amount of UV photons is provided by the AGN. The same is true in more sophisticated mixedmedium models (Binette et al. 1996, 1997). The observations seem to fit well the behavior of a model material shocked by the radio jet (Dopita \& Sutherland 1995). A shock velocity of $200 \mathrm{~km} \mathrm{~s}^{-1}$ can explain the ratio of the lines included in the $R$-band filter to the [O III] $\lambda 5007$ line emission.

Therefore, from the morphology (location of the radio jet) and these physical arguments (the flux measured being consistent with emission from shocked gas), we conclude that the ENLR structures of $3 \mathrm{C} 244.1$ are the result of the interaction of the radio jet with ISM gas.

C. F. acknowledges support from the STScI visitor program. We are very grateful to $\mathrm{P}$. Leahy for providing the radio map.
Allen, M. G. 1998, Ph.D. thesis, Australian National University, Canberra Allen, M. G., Dopita, M. A., Tsevtanov, Z. I., \& Sutherland, R. S. 1999, ApJ, 511, 686

Aoki, K. Kosugi, G., Wilson, A. S. \& Yoshida, M. 1999, ApJ, 521, 565

Axon, D. J., Marconi, A., Capetti, A., Macchetto, F. D., Schreier, E., \& Robinson, A. 1998, ApJ, 496, L75

Bennett, A. S. 1962a, MNRAS, 125, 75

.1962b, MmRAS, 68, 163

Binette, L., Wilson, A. S., Raga, A., \& Storchi-Bergmann, T. 1997, A\&A, 327,909

Binette, L., Wilson, A. S., \& Storchi-Bergmann, T. 1996, A\&A, 312, 365

Biretta, J. A., et al. 1996, WFPC2 Instrument Handbook, Version 4.0 (Baltimore: STScI)

Capetti, A., Axon, D. J., Kukula, M., Macchetto, D. F., Pedlar, A., Sparks, W. B., \& Boksenberg, A. 1995a, ApJ, 454, L85

Capetti, A., Axon, D. J., \& Macchetto, F. D. 1997, ApJ, 487, 560

Capetti, A., Axon, D. J., Macchetto, F. D., Sparks, W. B., \& Boksenberg, A. 1996, ApJ, 469, 554

Capetti, A., Macchetto, F. D., Axon, D. J., Sparks, W. B., \& Boksenberg, A. 1995 b, ApJ, 448, 600

de Koff, S., Baum, S. A., Sparks, W. B., Biretta, J., Golombek, D., Macchetto D. F., McCarthy, P., \& Miley G. K. 1996, ApJS, 107, 621

de Vries, W. H., et al. 1997, ApJS, 110, 191

Dopita, M. A., \& Sutherland, R. 1995, ApJ, 455, 468

. 1996, ApJS, 102, 161
Evans, I. N., Ford, H. C., Kinney, A. L., Antonucci, R. R. J., Armus, L., \& Caganoff, S. 1991, ApJ, 369, L27

Feinstein, C., Macchetto, F. D, Martel, A. R., \& Sparks, W. B. 1999, ApJ, 526,623

Ferland, G. J., \& Netzer, H. 1983, ApJ, 264, 105

Fernini, I., Burns, J. O., \& Perley, R. 1997, AJ, 114, 2292

Kukula, M. J., Ghosh, T., Pedlar, A., \& Schilizzi, R. T. 1999, ApJ, 518, 117

Martel, A. R., Sparks, W. B., Macchetto, F. D., Baum, S. A., Biretta, J. A., Golombek D., McCarthy, P. J., de Koff, S., \& Miley, G. K. 1998, AJ, 115, 1348

Martel, A.R., et al. 1999, ApJS, 122, 81

McCarthy, P. J., Miley, G. K., de Koff, S., Baum, S. A., Sparks , W. B., Golombek, D., Biretta, J., \& Macchetto, F. 1997, ApJS, 112, 415

McCarthy, P. J., Spinrad, H., \& Van Breugel, P. J. 1995, ApJS, 99, 27

Rawlings, S., Saunders, R., Eales, S. A., \& Mackay, C. D. 1989, MNRAS, 240, 701

Rönnback, J., Van Groningen, E., Wanders, I., \& Örndahl, E. 1996, MNRAS, 283, 282

Spinrad, H., Marr, J., Aguilar, L., \& Djorgovski, S. 1985, PASP, 97, 932

Taylor, D., Dyson, J., \& Axon, D. J. 1992, MNRAS, 255, 351

Wilson, A. S., Braatz, J. A., Heckman, T. M., Krolik, J. H., \& Miley, G. K. 1993, ApJ, 419, L61

Winge, C., Axon, D. J., Macchetto, F. D., \& Capetti, A. 1997, ApJ, 487, L121 